\title{
On series, integrals and continued fractions, III
}

\author{
by
}

\section{K. RAmachandra (Bangalore)}

1. Introduction. In [4] and [5] I proved some results and promised to prove some results on the summation of series involving $H_{n}=1+\frac{1}{2}+\ldots+\frac{1}{n}$. Some samples from [4] and [5] are

$$
\begin{aligned}
& \sum_{n=1}^{\infty} 2^{-n} H_{n}^{3}=\zeta(3)+\frac{1}{3}\left[\pi^{2} \log 2+(\log 2)^{3}\right], \\
& \sum_{n=1}^{\infty}(-1)^{n} n^{-1} H_{n}^{3}=\frac{9}{8} \zeta(3) \log 2+\frac{1}{4}(\log 2)^{4}-\frac{1}{8}(\pi \log 2)^{2}-\frac{\pi^{4}}{144}, \\
& \sum_{n=1}^{\infty}(-1)^{n}(3 n+1) 2^{-n} H_{n}^{3}=(\log 3-\log 2)^{2}, \\
& \sum_{n=1}^{\infty} n^{-1}(n+1)^{-1} H_{n}^{3}=\frac{\pi^{4}}{9}, \\
& \sum_{n=1}^{\infty} n 2^{-n-1} H_{n}^{4}=\frac{15}{4} \zeta(3)+\frac{13}{6} \pi^{2} \log 2+\frac{7}{3}(\log 2)^{3} .
\end{aligned}
$$

These with some additions were proved by myself and R. Sitaramachandrarao in [6]. However summations involving higher powers of $H_{n}$ promised in [4] and [5] have not been published so far. It is the object of this note to prove these results. More generally we start with any sequence $\left\{b_{n}\right\} \quad(n=$ $0,1,2, \ldots)$ of complex numbers and obtain in Section 2 , a general method of attacking summations of series involving

$$
G_{n}=b_{0}+b_{1}+\ldots+b_{n} .
$$

We reduce the summation of series like

$$
\sum_{n=1}^{\infty} f(n) G_{n}^{k}
$$

2000 Mathematics Subject Classification: 11-XX, 11Mxx, 11M99. 
where $k \geq 1$ and $f(n)(n=1,2, \ldots)$ is any sequence of complex numbers (subject to the convergence of (2)) to the summation of series like

$$
\sum_{n=1}^{\infty} R_{n}
$$

where $R_{n}=R_{n}(k)$ is a nice function. In particular it will turn out that $R_{n}$ is a rational function of $n$ in the special case $b_{0}=0, b_{1}=1, b_{2}=1 / 2, \ldots, b_{n}=$ $1 / n$ (i.e. $G_{n}=H_{n}$ ), provided $f(n)$ is a suitable rational function of $n$. Moreover it will turn out that

$$
\sum_{n=1}^{\infty} f(n) H_{n}^{k} \quad(k \geq 1 \text { is any integer })
$$

is a rational number for plenty of non-trivial choices of the sequence $\{f(n)\}$. In Section 3 we deal with some illustrative special cases and state Theorems 2 and 3. In Section 4 we give the evaluation of a series involving Euler's constant $\gamma$ (Theorem 4). In Section 5 we deduce from Theorem 1 a general result of some interest (Theorem 5). The referee has kindly pointed out that Theorem 3 can also be proved by using an important result [1] on Hadamard's product (a result which will be stated in a precise form in Section 6).

2. A key identity. A fundamental identity needed for our purposes is given by the following theorem.

TheOREM 1. Let $k \geq 1$ be any integer and $x, x_{1}, \ldots, x_{k}$ be any $k+1$ non-zero complex numbers such that $x_{i} \neq x_{j}$ whenever $i \neq j$. Then

$$
\begin{aligned}
x^{k}+\left\{\sum_{l=1}^{k}(x+\right. & \left.x_{l}\right)^{k}(-1)^{l} x_{l}^{-1} \\
& \left.\times\left(\prod_{l>j \geq 1}\left(x_{l}-x_{j}\right)^{-1}\right)\left(\prod_{k \geq i>l}\left(x_{i}-x_{l}\right)^{-1}\right)\right\} x_{1} \ldots x_{k} \\
= & (-1)^{k} x_{1} \ldots x_{k} .
\end{aligned}
$$

REMARK 1. In an earlier draft of this paper Theorem 1 was proved by a somewhat complicated method. It consisted in determining $A_{1}, \ldots, A_{k}$ and $D_{k}$ (all of which are independent of $x$ ) such that

$$
x^{k}+A_{1}\left(x+x_{1}\right)^{k}+\ldots+A_{k}\left(x+x_{k}\right)^{k}=D_{k} .
$$

Thanks are due to my friend C. R. Praneshachar, who later gave a very simple proof of Theorem 1. I will reproduce his proof after Remark 2. Both of us jointly will publish further proliferations of his idea in a forthcoming paper [3]. 
REMARK 2. The referee has pointed out an illuminating lemma which we state here. Let $X, X_{1}, \ldots, X_{k+1}$ be indeterminates and $P(X)=$ $\prod_{1 \leq j \leq k+1}\left(X-X_{j}\right)$. Then

$$
\sum_{1 \leq j \leq k+1}\left(P^{\prime}\left(X_{j}\right)\right)^{-1}\left(X-X_{j}\right)^{k}=(-1)^{k} .
$$

This with $X_{j}=-x_{j}(1 \leq j \leq k), X_{k+1}=0$ and $X=x$ gives Theorem 1 since $P^{\prime}\left(X_{k+1}\right)=x_{1} \ldots x_{k}$.

Proof of Theorem 1. Let $y$ be a complex variable. We decompose

$$
\frac{y^{k}}{\left(y-x_{1}\right) \ldots\left(y-x_{k}\right)}
$$

into partial fractions to obtain

$$
\frac{y^{k}}{\left(y-x_{1}\right) \ldots\left(y-x_{k}\right)}=1+\sum_{j=1}^{k} \frac{1}{y-x_{j}} \cdot \frac{x_{j}^{k}}{\prod_{i \neq j}\left(x_{j}-x_{i}\right)} .
$$

Here we put $y=1$ and replace $x_{j}$ by $x_{j} x^{-1}+1$. We obtain

$$
\frac{1}{\prod_{j=1}^{k}\left(1-\left(x_{j} x^{-1}+1\right)\right)}=1+\sum_{j=1}^{k} \frac{1}{\left(1-\left(x_{j} x^{-1}+1\right)\right)} \cdot \frac{\left(x_{j} x^{-1}+1\right)^{k}}{\prod_{i \neq j}\left(x_{j} x^{-1}-x_{i} x^{-1}\right)},
$$

i.e.

$$
\frac{(-x)^{k}}{x_{1} \ldots x_{k}}=1-\sum_{j=1}^{k} \frac{x}{x_{j}} \cdot \frac{\left(x+x_{j}\right)^{k} x^{-k}}{x^{-k+1}} \prod_{i \neq j}\left(x_{j}-x_{i}\right)^{-1}
$$

i.e.

$$
(-1)^{k} x^{k}+\sum_{l=1}^{k} \frac{x_{1} \ldots x_{k}}{x_{l}}(-1)^{k-l} \frac{\left(x+x_{l}\right)^{k}}{\prod_{j<l}\left(x_{l}-x_{j}\right) \prod_{j>l}\left(x_{j}-x_{l}\right)}=x_{1} \ldots x_{k} .
$$

Multiplying throughout by $(-1)^{k}$ we get Theorem 1 .

3. Some applications of Theorem 1. We first illustrate our method of applying Theorem 1 by considering some special cases and finally we are led to Theorem 4 which will be stated at the end of this section.

(a) We put $k=1$ in Theorem 1 . We get

$$
x-\left(x+x_{1}\right)=-x_{1} .
$$

In (1) we consider the case $b_{0}=0, b_{n}=1 / n(n=1,2, \ldots)$. Obviously $G_{n}=H_{n}$. We have (from (5) with $x=H_{n}$ and so with $x_{1}=1 /(n+1)$ )

$$
F(n)\left(H_{n+1}-H_{n}\right)=\frac{F(n)}{n+1}
$$


for any sequence $F(n)(n=1,2, \ldots)$. Summing up from $n=1$ to $\infty$ we have (subject to convergence of the series involved)

$$
\sum_{n=1}^{\infty} F(n) H_{n+1}-\sum_{n=1}^{\infty} F(n) H_{n}=\sum_{n=1}^{\infty} F(n)(n+1)^{-1} .
$$

Here the left hand side is nothing but

$$
\sum_{n=1}^{\infty} F(n) H_{n+1}-F(1) H_{1}-\sum_{n=1}^{\infty} F(n+1) H_{n+1} .
$$

This with (7) gives

$$
\sum_{n=1}^{\infty} H_{n+1}(F(n)-F(n+1))-F(1) H_{1}=\sum_{n=1}^{\infty} F(n)(n+1)^{-1} .
$$

Transposing we obtain

$$
\sum_{n=1}^{\infty} H_{n+1}(F(n)-F(n+1))=F(1) H_{1}+\sum_{n=1}^{\infty} F(n)(n+1)^{-1} .
$$

Equation (9) converts the problem of summing up

$$
\sum_{n=1}^{\infty} H_{n+1}(F(n)-F(n+1))
$$

to one of $\sum_{n=1}^{\infty} F(n)(n+1)^{-1}$ (which is usually much simpler). For example when $F(n)=(n+1) 2^{-n}$ it follows that (10) is a rational number. Certainly we can take $F(n)$ to be $(n+1) 2^{-n} \phi(n)$ where $\phi(n)$ is any polynomial in $n$ with integer coefficients.

(b) We put $k=2$ in Theorem 1 . We get $x^{2}-\left(x+x_{1}\right)^{2} x_{1}^{-1}\left(x_{2}-x_{1}\right)^{-1} x_{1} x_{2}+\left(x+x_{2}\right)^{2} x_{2}^{-1}\left(x_{2}-x_{1}\right)^{-1} x_{1} x_{2}=x_{1} x_{2}$, i.e.

$$
x^{2}\left(x_{2}-x_{1}\right)-\left(x+x_{1}\right)^{2} x_{2}+\left(x+x_{2}\right)^{2} x_{1}=x_{1} x_{2}\left(x_{2}-x_{1}\right) .
$$

Putting $x_{1}=a, x_{2}=a+b$ (where $a$ and $b$ are any two complex numbers) we have

$$
b x^{2}-(a+b)(x+a)^{2}+a(x+a+b)^{2}=a b(a+b) .
$$

This gives (with $x=H_{n}, a=1 /(n+1)$ and $b=1 /(n+2)$ ),

$$
\frac{H_{n}^{2}}{n+2}-\left(\frac{1}{n+1}+\frac{1}{n+2}\right) H_{n+1}^{2}+\frac{1}{n+1} H_{n+2}^{2}=\frac{2 n+3}{(n+1)^{2}(n+2)^{2}} .
$$

Multiplying throughout by $2^{-n}(n+1)^{2}(n+2)^{2}$ (we can multiply this by a further function $\phi(n)$ which is any polynomial in $n$ with integer coefficients), 
we obtain

$$
\begin{aligned}
H_{n}^{2}(n+1)^{2}(n+2) 2^{-n}- & H_{n+1}^{2}(2 n+3)(n+1)(n+2) 2^{-n} \\
& +H_{n+2}^{2}(n+1)(n+2)^{2} 2^{-n}=(2 n+3) 2^{-n} .
\end{aligned}
$$

We sum up from $n=1$ to $\infty$ and obtain

$$
\begin{aligned}
\sum_{n=1}^{\infty} H_{n}^{2}(n+1)^{2}(n+2) & 2^{-n}-\sum_{n=1}^{\infty} H_{n}^{2}(2 n+1)(n)(n+1) 2^{-n+1}+6 \\
+ & \sum_{n=1}^{\infty} H_{n}^{2}(n-1) n^{2} 2^{-n+2}-9=\sum_{n=1}^{\infty}(2 n+3) 2^{-n} .
\end{aligned}
$$

This gives

THEOREM 2. We have

$$
\sum_{n=1}^{\infty} \phi_{2}(n) H_{n}^{2} 2^{-n}=\sum_{n=0}^{\infty}(2 n+3) 2^{-n}
$$

(with $\left.\phi_{2}(n)=(n-1)\left(n^{2}-5 n-2\right)\right)$, which can be easily seen to be a rational number.

REMARK. We have plenty of choices (in place of $\left.\phi_{2}(n)\right)$ where $\phi_{2}(n)$ can be easily replaced by many non-trivial polynomials in $n$ (by choosing $\phi(n)$ occurring after (12) suitably.)

(c) Many generalizations are clear. We can certainly take $k$ to be any positive integer. For example taking $k=3$ in Theorem 1 , we get

$$
\begin{aligned}
x^{3}+\{ & -\left(x+x_{1}\right)^{3} x_{1}^{-1}\left(x_{3}-x_{1}\right)^{-1}\left(x_{2}-x_{1}\right)^{-1} \\
& +\left(x+x_{2}\right)^{3} x_{2}^{-1}\left(x_{2}-x_{1}\right)^{-1}\left(x_{3}-x_{2}\right)^{-1} \\
& \left.-\left(x+x_{3}\right)^{3} x_{3}^{-1}\left(x_{3}-x_{1}\right)^{-1}\left(x_{3}-x_{2}\right)^{-1}\right\} x_{1} x_{2} x_{3}=-x_{1} x_{2} x_{3},
\end{aligned}
$$

i.e.

$$
\begin{aligned}
x^{3}\left(x_{3}-x_{1}\right)\left(x_{3}-\right. & \left.x_{2}\right)\left(x_{2}-x_{1}\right)-\left(x+x_{1}\right)^{3} x_{2} x_{3}\left(x_{3}-x_{2}\right) \\
& +\left(x+x_{2}\right)^{3} x_{1} x_{3}\left(x_{3}-x_{1}\right)-\left(x+x_{3}\right)^{3} x_{1} x_{2}\left(x_{2}-x_{1}\right) \\
= & -x_{1} x_{2} x_{3}\left(x_{3}-x_{1}\right)\left(x_{3}-x_{2}\right)\left(x_{2}-x_{1}\right) .
\end{aligned}
$$

We put $x_{1}=a, x_{2}=a+b, x_{3}=a+b+c$, where $a, b, c$ are any complex numbers. We obtain

$$
\begin{aligned}
x^{3}(b+c)(c)(b)-(x+a)^{3}(a+ & b)(a+b+c)(c) \\
+ & (x+a+b)^{3}(a)(a+b+c)(b+c) \\
& -(x+a+b+c)^{3}(a)(a+b)(b) \\
= & -a b c(a+b)(b+c)(a+b+c) .
\end{aligned}
$$


Here we can put $x=H_{n}, a=1 /(n+1), b=1 /(n+2), c=1 /(n+3)$ and proceed as before. We conclude that

$$
\sum_{n=1}^{\infty} \phi_{3}(n) 2^{-n} H_{n}^{3}
$$

is a rational number for infinitely many non-trivial polynomials $\phi_{3}(n)$ with integer coefficients.

(d) Just as we worked with $k=1,2$ and 3 we can work with $k=4,5,6, \ldots$ We obtain the following theorem.

TheOREM 3. Let $k \geq 1$ be any fixed integer. Then for a non-trivial infinite class of polynomials $\phi_{k}(n)($ in $n)$ with integer coefficients, the series

$$
\sum_{n=1}^{\infty} \phi_{k}(n) 2^{-n} H_{n}^{k}
$$

is a rational number.

4. Series evaluations involving Euler's constant $\gamma$. We next consider

$$
b_{0}=-\gamma \quad \text { and } \quad b_{n}=\frac{1}{n}-\log \left(\frac{n+1}{n}\right) \quad(n=1,2, \ldots) .
$$

Now

$$
G_{n}=-\gamma+\sum_{m=1}^{n} \frac{1}{m}-\log (n+1) .
$$

We are led to series involving higher powers of $G_{n}$. To illustrate our method we consider the special case $k=2$ of Theorem 1 . We go back to the identity (12) (which is a special case of Theorem 1). Here we put $x=G_{n}, a=b_{n+1}$ and $b=b_{n+2}$. This gives

$$
G_{n}^{2} b_{n+2}-\left(b_{n+1}+b_{n+2}\right) G_{n+1}^{2}+b_{n+1} G_{n+2}^{2}=\left(b_{n+1}+b_{n+2}\right) b_{n+1} b_{n+2} .
$$

Note that $G_{n}=O\left(n^{-1}\right)$ and $b_{n}=O\left(n^{-2}\right)$. We now sum up (15) from $n=1$ to $\infty$. We obtain

$$
\begin{aligned}
G_{1}^{2} b_{3}+G_{2}^{2} b_{4}+\sum_{n=1}^{\infty} G_{n+2}^{2} b_{n+4}-\left(b_{2}+b_{3}\right) G_{2}^{2} & \\
& -\sum_{n=1}^{\infty}\left(b_{n+2}+b_{n+3}\right) G_{n+2}^{2}+\sum_{n=1}^{\infty} b_{n+1} G_{n+2}^{2} \\
& =\sum_{n=1}^{\infty} b_{n+1} b_{n+2}\left(b_{n+1}+b_{n+2}\right) .
\end{aligned}
$$


This leads to the identity (which is not neat but our method leads to a host of other identities) which we state as Theorem 5 .

Theorem 4. Let $\gamma$ be the limit as $n \rightarrow \infty$ of $H_{n}-\log n$. Put

$$
G_{n}=-\gamma+\sum_{m=1}^{n}\left(\frac{1}{m}-\log \frac{m+1}{m}\right) .
$$

Then

$$
\begin{aligned}
\sum_{n=3}^{\infty} & \left\{\frac{1}{n(n-1)}-\frac{1}{(n+1)(n+2)}+\log \left(1-\frac{4}{n^{3}+3 n^{2}}\right)\right\} G_{n}^{2} \\
& +\gamma^{2}\left(-\frac{1}{4}+\log \frac{6}{5}\right) \\
& -2 \gamma\left\{(1-\log 2)\left(\frac{1}{3}-\log \frac{4}{3}\right)+\left(\frac{3}{2}-\log 3\right)\left(\log \frac{8}{5}-\frac{7}{12}\right)\right\} \\
& +(1-\log 2)^{2}\left(\frac{1}{3}-\log \frac{4}{3}\right)+\left(\frac{3}{2}-\log 3\right)^{2}\left(\log \frac{8}{5}-\frac{7}{12}\right) \\
= & \sum_{n=1}^{\infty}\left(\frac{1}{n+1}-\log \frac{n+2}{n+1}\right)\left(\frac{1}{n+2}-\log \frac{n+3}{n+2}\right) \\
& \times\left(\frac{1}{n+1}+\frac{1}{n+2}-\log \frac{n+3}{n+1}\right) .
\end{aligned}
$$

Remark. Certainly we can get series evaluation involving $G_{n}^{k}(k=$ $3,4,5, \ldots)$.

5. A general result on $G_{n}^{k}$. Theorem 1 certainly gives the identity

$$
x^{k}+A_{1}\left(x+x_{1}\right)^{k}+\ldots+A_{k}\left(x+x_{k}\right)^{k}=D_{k}
$$

where $A_{1}, \ldots, A_{k}$ and $D_{k}$ are all independent of $x$.

We now explain how to apply Theorem 1 to the summation of (2). We choose $x=b_{0}$ and

$$
x_{1}=b_{n+1}, \quad x_{2}=b_{n+1}+b_{n+2}, \ldots, x_{k}=b_{n+1}+b_{n+2}+\ldots+b_{n+k} .
$$

We see, with $A_{0}=1$ and $A_{1}, \ldots, A_{k}$ and $D_{k}$, that these depend only on $b_{n+1}, \ldots, b_{n+k}$. For a fixed $k$ and any fixed sequence $F(1), F(2), \ldots$ we write

$$
\begin{aligned}
C_{0}(n) & =F(n) A_{0}, \quad C_{1}(n)=F(n) A_{1}, \ldots, C_{k}(n)=F(n) A_{k}, \\
R(n) & =D_{k}(n) F(n) .
\end{aligned}
$$

Then subject to the convergence condition (and plainly we need $x_{i} \neq x_{j}$ for 
$i \neq j$ ) we have the identity

$$
\sum_{n=1}^{\infty} C_{0}(n) G_{n}^{k}+\sum_{n=1}^{\infty} C_{1}(n) G_{n+1}^{k}+\ldots+\sum_{n=1}^{\infty} C_{k}(n) G_{n+k}^{k}=\sum_{n=1}^{\infty} R(n) .
$$

Here the left hand side is

$$
\begin{aligned}
\left(\sum_{n=1}^{k}\right. & \left.C_{0}(n) G_{n}^{k}+\sum_{n=1}^{\infty} C_{0}(n+k) G_{n+k}^{k}\right) \\
& +\left(\sum_{n=1}^{k-1} C_{1}(n) G_{n+1}^{k}+\sum_{n=1}^{\infty} C_{1}(n+k-1) G_{n+k}^{k}\right) \\
& +\ldots+\left(\sum_{n=1}^{1} C_{k-1}(n) G_{n+k-1}^{k}+\sum_{n=1}^{\infty} C_{k-1}(n+1) G_{n+k}^{k}\right) \\
& +\sum_{n=1}^{\infty} C_{k}(n) G_{n+k}^{k} \\
= & \sum_{n=1}^{k} C_{0}(n) G_{n}^{k}+\sum_{n=1}^{k-1} C_{1}(n) G_{n+1}^{k}+\ldots+\sum_{n=1}^{1} C_{k-1}(n) G_{n+k-1}^{k} \\
& +\sum_{n=1}^{\infty}\left(C_{0}(n+k)+C_{1}(n+k-1)+C_{2}(n+k-2)\right. \\
& \left.+\ldots+C_{k}(n)\right) G_{n+k}^{k} .
\end{aligned}
$$

Writing

$$
f(n+k)=C_{0}(n+k)+C_{1}(n+k-1)+C_{2}(n+k-2)+\ldots+C_{k}(n)
$$

we have the following theorem.

THEOREM 5. In the notation explained above, we have

$$
\sum_{n=1}^{\infty} f(n+k) G_{n+k}^{k}
$$

$=\sum_{n=1}^{\infty} R(n)-\left\{\sum_{n=1}^{k} C_{0}(n) G_{n}^{k}+\sum_{n=1}^{k-1} C_{1}(n) G_{n+1}^{k}+\ldots+\sum_{n=1}^{1} C_{k-1}(n) G_{n+k-1}^{k}\right\}$

and plainly $\sum_{n=1}^{\infty} f(n) G_{n}^{k}$ equals the left hand side of (22) plus the finite $\operatorname{sum} \sum_{n=1}^{k} f(n) G_{n}^{k}$.

6. Concluding remarks and acknowledgements. The author is indebted to the referee for pointing out the following theorem (see [1]). 
TheOREM 6. Let

$$
g_{1}(x)=\sum_{n=1}^{\infty} a_{n} x^{n} \quad \text { and } \quad g_{2}(x)=\sum_{n=1}^{\infty} b_{n} x^{n}
$$

be two formal power series with coefficients in a commutative field $K$. Define the Hadamard product of $g_{1}(x)$ and $g_{2}(x)$ by the equation

$$
\left(g_{1} * g_{2}\right)(x)=\sum_{n=1}^{\infty} a_{n} b_{n} x^{n} .
$$

If $g_{1}(x)$ and $g_{2}(x)$ satisfy a linear differential equation with coefficients in $K[x]$, the same also holds for $\left(g_{1} * g_{2}\right)(x)$.

REMARK 1. Note that

$$
h_{1}(x)=\sum_{n=1}^{\infty} H_{n} x^{n}=-(\log (1-x))(1-x)^{-1}
$$

satisfies the differential equation $(1-x)\left((1-x) h_{1}(x)\right)^{\prime}=1$. Thus Theorem 6 implies that the $k$ th Hadamard product

$$
\sum_{n=1}^{\infty} H_{n}^{k} x^{n}
$$

satisfies a linear differential equation with coefficients in $\mathbb{Q}[x], \mathbb{Q}$ being the rational number field. Hence Theorem 6 certainly implies Theorem 3 .

REMARK 2. It must be mentioned that series involving $H_{n}$ have recently been considered by some other authors. See for example [2] which certainly deserves to be mentioned here.

Acknowledgements. The author is indebted to the referee for pointing out Theorem 6 and also the paper mentioned in Remark 2 above. When this work was in progress the author was a Senior Scientist of the INSA. The author is very much thankful to Professor Raja Ramanna for encouragement. Also the author wishes to thank Mrs. J. N. Sandhya and Kumari Pramila for the technical help.

\section{References}

[1] L. Comtet, Analyse Combinatoire, Tome 1, Presses Univ. France, 1970, exercise 30, p. 97.

[2] O. M. Ogreid and P. Osland, Summing one- and two-dimensional series related to the Euler series, J. Comput. Appl. Math. 98 (1998), 245-271.

[3] C. R. Praneshachar and K. Ramachandra, A new identity - III, to appear.

[4] K. Ramachandra, On the summation of certain series involving $H_{n}=1+\frac{1}{2}+\ldots+\frac{1}{n}$, in: Proc. 45th Indian Science Congress (Madras, 1958), Math. Abstract No. 3. 
[5] K. Ramachandra, On series, integrals and continued fractions - I, Hardy-Ramanujan J. 4 (1981), suppl., 1-11.

[6] K. Ramachandra and R. Sitaramachandrarao, On series, integrals and continued fractions - II, Madras Univ. J. Sect. B 51 (1988), 181-198.

Nat. Inst. of Adv. Studies

TIFR Centre

I. I. Sc. Campus

I. I. Sc. Campus

Bangalore-560012, India P.O. Box 1234

Bangalore-560012, India

E-mail: kram@math.tifrbng.res.in

Received on 7.1.2000

and in revised form on 1.12.2000 